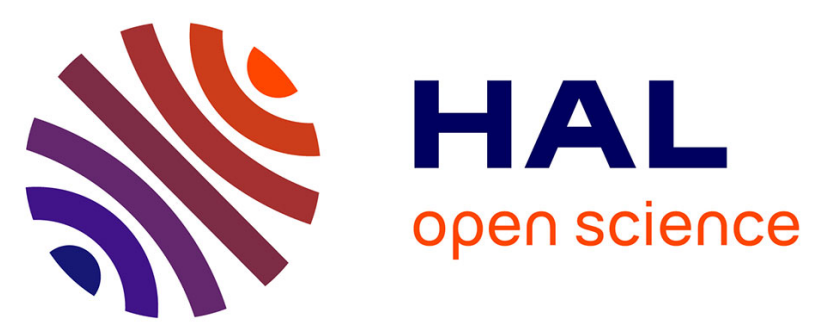

\title{
Double fluorescent knock-in mice to investigate endogenous mu-delta opioid heteromer subscellular distribution.
}

Lyes Derouiche, Stéphane Ory, Dominique Massotte

\section{- To cite this version:}

Lyes Derouiche, Stéphane Ory, Dominique Massotte. Double fluorescent knock-in mice to investigate endogenous mu-delta opioid heteromer subscellular distribution.. Receptor-Receptor Interactions in the Central Nervous System., 140, 2018, Neuromethods, 10.1007/978-1-4939-8576-0_10 . hal-03192409

\author{
HAL Id: hal-03192409 \\ https://hal.science/hal-03192409
}

Submitted on 7 Apr 2021

HAL is a multi-disciplinary open access archive for the deposit and dissemination of scientific research documents, whether they are published or not. The documents may come from teaching and research institutions in France or abroad, or from public or private research centers.
L'archive ouverte pluridisciplinaire HAL, est destinée au dépôt et à la diffusion de documents scientifiques de niveau recherche, publiés ou non, émanant des établissements d'enseignement et de recherche français ou étrangers, des laboratoires publics ou privés. 
1 Title: Double fluorescent knock-in mice to investigate endogenous mu-delta opioid

2 heteromer subscellular distribution.

3

4 Authors: Lyes Derouiche, Stéphane Ory and Dominique Massotte

5

6 Address:

7 Institut des Neurosciences Cellulaires et Intégratives, UPR 3212 CNRS, Strasbourg, France

8 Corresponding author:

9 Dominique Massotte

10 INCI UPR 3212 CNRS

115 rue Blaise Pascal

$12 \quad$ F-67084 Strasbourg cedex 03

13 France

14 Tel : +0388456652

15 d.massotte@unistra.fr

16

17 Running title: Endogenous Mu-delta heteromer internalization

18

19 Key words: heteromers, opioid receptors, mu-delta, trafficking, primary neuronal culture, 20 GPCR

21

22 Number of words: 4943

23 Number of figures: 2

24 Number of tables: 1 


\section{ABSTRACT}

The heteromerization of $\mathrm{Mu}(\mathrm{MOP})$ and delta (DOP) opioid receptors has been extensively studied in heterologous systems. These studies demonstrated significant functional interaction of MOP and DOP evidenced by new pharmacological properties and intracellular signalling in transfected cells co-expressing the receptors. Due to the lack of appropriate tools for receptor visualization, such as specific antibodies, the pharmacological and functional properties of MOP-DOP heteromers in cells naturally expressing these receptors remains poorly understood. To address endogenous MOP-DOP heteromer trafficking and signalling in vivo and in primary neuronal cultures, we generated a double knock-in mouse line expressing functional fluorescent versions of DOP and MOP receptors. This mouse model has successfully been used to map the neuroanatomic distribution of the receptors and to identify brain regions in which the MOP-DOP heteromers are expressed. Here, we describe a method to quantitatively and automatically analyze changes in the subcellular distribution of MOP-DOP heteromers in primary hippocampal culture from this mouse model. This approach provides a unique tool to address specificities of endogenous MOPDOP heteromer trafficking.

\section{INTRODUCTION}

Opioid receptors belong to the subfamily of Class A G protein-coupled receptors (GPCRs). Four subtypes of opioid receptors mu (MOP), delta (DOP), kappa (KOP) and nociceptin (NOP) receptor respectively encoded by the OPRM1, OPRD1, OPRK1 and OPRL1 genes have been identified several decades ago (for review see [1-3]). These seven transmembrane domain receptors are functional in a monomeric form, but can also associate among themselves to generate a larger assembly or with different subtypes of opioids or non-opioid 
receptors. In the latter case, the new entity is called heteromer and may exhibit specific functional properties.

In the case of the opioid system, MOP and DOP functional interactions are well documented Among others, they are essential for the development of opiate tolerance $[4,5]$. Numerous studies indicate that co-expression of the two receptors in heterologous systems promotes the formation of MOP-DOP heteromers, which affects binding and signaling properties $[6,7]$. However, in spite of a growing body of evidence in favor of the presence of MOP-DOP heteromers in vivo, the molecular mechanisms underlying functional interactions between these two receptors remain poorly characterized[8]. This is mainly due to the lack of appropriate tools, especially specific antibodies.

To deal with this issue and study MOP-DOP heteromers in vivo, we generated a double fluorescent knock-in mouse line co-expressing DOP and MOP receptors respectively fused to their C-terminus to the enhanced green fluorescent protein (DOP-eGFP) or mcherry (MOPmcherry). The DOP-eGFP and MOP-mCherry functional fusions allow highly specific and simultaneous visualization of endogenously expressed receptors with subcellular resolution and proved to be unique tools for neuroanatomical studies [9]. Mapping of MOP and DOP receptors in the central and peripheral nervous systems indeed revealed MOP-DOP neuronal co-expression in discrete neuronal networks essential for survival such as the nociceptive pathway (see also mouse brain atlas at http://mordor.ics-mci.fr/). Specific targeting using the fluorescent tags also revealed MOP-DOP physical proximity in the hippocampus providing strong rationale for the existence of endogenous MOP-DOP heteromers [9]. In addition, the double fluorescent knock-in mice represent unique tools to explore the dynamics of this 
complex under physiological or pathological conditions and to characterize the functional impact of MOP-DOP heteromers in the central and peripheral nervous system.

In this chapter, we describe optimized conditions for visualization of endogenous MOP-DOP heteromers in primary hippocampal neurons obtained from the double fluorescent knock-in mice. We also provide a protocol for automatic quantitative analysis of confocal images with an open source software to determine changes in receptor subcellular localization. This method allowed MOP-DOP heteromers monitoring and specific determination of their intracellular fate upon pharmacological activation.

\section{MATERIAL AND METHODS}

\section{Animals}

Double knock-in mice co-expressing fluorescent DOP and MOP receptors were obtained by crossing previously generated DOP-eGFP and MOP-mcherry knock-in mice. Briefly, DOPeGFP knock-in mice expressing the delta opioid receptor fused to its C-terminus to the eGFP were generated by homologous recombination by inserting the eGFP cDNA into exon 3 of the delta opioid receptor gene, in frame and $5^{\prime}$ from the stop codon [10]. MOP-mcherry knock-in mice expressing the mu opioid receptor fused to its C-terminus to the red fluorescent protein mCherry were generated by homologous recombination following a procedure similar to the one used for DOR-eGFP knock-in mice [9]. The construct transfected into ES cells comprised a Gly-Ser-Ile-Ala-Thr linker followed by the cDNA sequence encoding the fluorescent protein (eGFP or mcherry). For subsequent clone selection, a resistance gene was included that corresponded to neomycin flanked by loxP sites for DOPeGFP or to hygromycin flanked by FRT sites for MOP-mcherry (Figure 1). The resistance gene was removed by microinjection of a plasmid expressing the recombinase. Blastocysts 
were implanted in pseudo gestant BalbC females. Chimeric mice were crossed with C57B16/J mice to obtain F1 heterozygous generation. Heterozygous animals were crossed to generate mice homozygous for Oprd1-eGFP or Oprm1-mcherry that are fertile and develop normally.

Double knock-in animals were obtained by crossing the single knock-in mouse lines. The genetic background of all mice was C57/BL6/J: 129svPas (50:50 \%).

Mice were housed in animal facility under controlled temperature $\left(21 \pm 2{ }^{\circ} \mathrm{C}\right)$ and humidity (45 $\pm 5 \%$ ) on a 12-h dark-light cycle with food and water ad libitum. All experiments were performed in accordance to the European legislation (directive 2010/63/EU acting on protection of laboratory animals) and the local ethical committee.

\section{Primary hippocampal culture}

\subsection{Material and reagents:}

- Double knock-in new born mice pups (P0-P3).

- $70 \%$ ethanol solution

- MilliQ water (autoclaved, or sterile filtered $0.22 \mu \mathrm{m}$ ).

- Borate buffer (see setup and procedures)

- Poly-L-lysine hydrobromide (Sigma cat. No. P2623)

- 13 mm coverslips (Sigma, cat. no. P6407) coated with Poly-L-lysine (see setup and procedure)

- 24-well sterile culture plates (Falcon cat. no. 353047).

- Pasteur pipets (flamed at the extremity, cotton plugged and autoclaved).

- Hibernate minus phenol red (BrainBits SKU: HAPR)

- Papain (Worthington, cat. no. LS003126) 
- Dulbecco's Modified Eagle's Medium (DMEM) with 4.5g glucose (GIBCO, cat. no. 71966-029)

- Neurobasal A (GIBCO, cat. no. A13710-01)

- Foetal Calf Serum FCS heat inactivated. Caution: test several batches to determine the best one for your culture conditions.

- Glutamax $^{\mathrm{TM}}$ (GIBCO, cat. no. 35050061)

- L-glutamine (GIBCO, cat. no. 25030081 )

- Penicillin-streptomycin (P/S) (cat. no. 15140122)

- DNAse (Sigma cat. no. DN25).

- B27 supplement (GIBCO, cat. no. 17504044)

- Trypan blue solution (Sigma, cat. no. T8154)

- Paraformaldehyde $32 \%$ solution diluted to $4 \%$ before use (see setup and reagents).

- Phosphate buffer saline (PBS). (Sigma-Aldrich, cat. no. P5493)

- Cell strainer $70 \mu \mathrm{m}$ (Falcon, cat. no. 352350)

\subsection{Setup and procedures}

2.2.1. Borate buffer: dissolve boric acid $1,24 \mathrm{~g}$ and Sodium tetraborate (borax) $1,9 \mathrm{~g}$ in $400 \mathrm{ml}$ MilliQ $\mathrm{H}_{2} \mathrm{O}$. $\mathrm{pH}$ should be 8.4 . Sterile filter $(0.22 \mu \mathrm{m})$ before use. Caution: borax is a hazardous substance, manipulate cautiously and eliminate waste according to the safety rules fixed by your institution/government.

2.2.2. Coverslips sterilisation and coating: put coverslips in a $100-\mathrm{mm}$ petri dish, sterilise in 70\% ethanol during $2 \mathrm{~h}$ under gentle agitation, let dry completely under laminar flow, and transfer to culture plates. Rinse once with MilliQ water. Coat coverslips with poly-L-lysine $25 \mu \mathrm{g} / \mathrm{ml}$ final concentration in borate buffer; incubate at $37^{\circ} \mathrm{C}$ for $2 \mathrm{~h}$ to overnight. Rinse 3 times with sterile water and pre-warm in DMEM medium. Coated coverslips may be 
prepared several days before use, dried in the laminar flow hood and kept sealed at $4^{\circ} \mathrm{C}$ for

141 up to 1 month.

2.2.3. Dissection medium: Prepare $5 \mathrm{~mL}$ of of ice cold Hibernate supplemented with $1 \mathrm{X} P / \mathrm{S}$ and $0.5 \mathrm{nM}$ glutamax per animal and transfer $0.5 \mathrm{~mL}$ dissection medium in a $15-\mathrm{mL}$ centrifugation tube per animal (2 hippocampi).

2.2.4. Enzyme solution: prior to dissection, prepare a fresh solution of Papain at $40 \mathrm{U} / \mathrm{mL}$ concentration in Hibernate medium, incubate 5 minutes at $37^{\circ} \mathrm{C}$ in a water bath then keep on ice until use. Prepare $0.5 \mathrm{~mL}$ per animal (2 hippocampi).

2.2.5. Plating medium: Prepare $12 \mathrm{~mL}$ DMEM medium supplemented with $4.5 \mathrm{~g} / \mathrm{l}$ glucose $+10 \%$ heat inactivated FCS $+2 \mathrm{mM}$ Glutamine + Pen/strep) per animal on the day of use.

2.2.6. Growing medium: Prepare $12 \mathrm{~mL}$ Neurobasal medium supplemented with $2 \%$ B27, 2mM glutamax, $0.5 \mathrm{mM}$ glutamine and $1 \mathrm{X} \mathrm{P} / \mathrm{S}$ per animal on the day of use.

2.2.7. Phosphate-buffered saline (PBS): prepare 1L 1X PBS working solution from 10X stock solution by diluting with MilliQ water. Check the $\mathrm{pH}$ and adjust to 7.4 with $1 \mathrm{M} \mathrm{HCl}$ or $1 \mathrm{M} \mathrm{NaOH}$ solutions if needed. Sterile filter $0.22 \mu \mathrm{m}$ and keep at $4^{\circ} \mathrm{C}$ for up to 6 months.

\subsubsection{Fixation solution: dilute paraformaldehyde (PFA) $32 \%$ solution to $4 \%$ final} concentration in PBS 0.1M, adjust $\mathrm{pH}$ to 7.4 if needed. Prepare $500 \mu \mathrm{l}$ per well for use in 24well plate. Keep up to 5 days at $4^{\circ} \mathrm{C}$ and up to 6 months at $-20^{\circ} \mathrm{C}$. Caution: PFA a hazardous highly toxic substance, manipulate under flow hood and eliminate waste according to the safety rules fixed by your institution/government.

\subsection{Dissection and culture procedures}

\subsubsection{Dissection and cell dissociation}

Decapitate pups. Transfer the head in a $33 \mathrm{~mm}$ petri dish with $1.5 \mathrm{~mL}$ ice cold dissection medium and isolate the brain. Place the isolated brain in a new $33 \mathrm{~mm}$ petri dish with $1.5 \mathrm{~mL}$ 
ice cold dissection medium. Remove the meninges, dissect to isolate the two hippocampi and keep them in $0.5 \mathrm{~mL}$ dissection solution in a $15 \mathrm{~mL}$-tube in ice. Add $0.5 \mathrm{~mL}$ of papain solution per tube to $0.5 \mathrm{~mL}$ dissection medium ( $1 \mathrm{~mL}$ final / 2 hippocampi). Place tubes in a water bath at $37^{\circ} \mathrm{C}$ for 30 minutes with gentle shaking every 5-10 minutes. 5-10 minutes before digestion ends, add DNase at a final concentration of $1 \mathrm{mg} / \mathrm{mL}$.

Remove papain solution by decantation, add $1 \mathrm{~mL}$ of Hibernate, and triturate with Pasteur pipet until the tissue is completely dissociated (about 15 to 20 times up-and-down are sufficient) (see note 1). Centrifuge at $1000 \mathrm{~g}$ for 5 minutes at $22^{\circ} \mathrm{C}$. Remove the supernatant, add $1 \mathrm{~mL}$ plating medium to resuspend cells (3 to 5 gentle up-and-down with Pasteur pipet). Filter the cell suspension through a $70 \mu \mathrm{m}$-cell strainer to remove any residual aggregates. Count cells by diluting $20 \mu 1$ of cell suspension in $80 \mu 1$ of 1:10 Trypan blue solution diluted in PBS. Place $20 \mu \mathrm{l}$ of this solution in a cell counting chamber and count cells excluding Trypan blue (viable cells) only.

\subsubsection{Plating and feeding}

Prewarm poly-L-Lysine coated plates in DMEM medium at $37^{\circ} \mathrm{C}$. Remove DMEM and plate cells in 24-well plates at a density of 80000 to 100000 cells per well in a final volume of 500 $\mu \mathrm{L}$.

Incubate in a humid incubator at $37^{\circ} \mathrm{C}$ and $5 \% \mathrm{CO} 2$ and allow cells to adhere to the bottom of plates during one hour. Remove the plating medium by aspiration, rinse once with $500 \mu$ of prewarmed Neurobasal A medium, then add $500 \mu$ l of growing medium and return back to the incubator. Let cells grow during at least twelve days with half of the medium replaced every four to five days.

\section{Processing and pharmacological treatments}


Pharmacological treatments should be realized between DIV 12 and DIV 15 after plating (see

188

189

190

191

192

193

194

note 2). Ligands are added in a volume not exceeding $10 \%$ of the culture medium volume.

Incubate cells during the appropriate time. At the end of the pharmacological treatment, remove the plate from the incubator and immediately place on ice, carefully aspirate the medium and wash twice with ice cold sterile filtered PBS. Remove PBS and add 500 $\mu$ L of PFA 4\% in ice cold PBS and incubate during 20 minutes on ice. Remove PFA and rinse twice with ice cold PBS and proceed to immunostaining or keep sealed with Parafilm in $500 \mu \mathrm{L}$ PBS at $4^{\circ} \mathrm{C}$ up to 30 days (see note 3 ).

\section{Immunocytofluorescence (ICF):}

\subsection{Material and reagents}

- $\quad$ Phosphate buffer Saline 0.1M, pH 7.4.

- Normal Goat Serum (NGS) (Sigma cat. no. S26)

- Tween20 (Euromedex cat. no. 2001-B)

- Primary and secondary antibodies (see table 1)

- $\quad$ ProLong ${ }^{\mathrm{TM}}$ Gold Antifade Mounting medium (Molecular Probes cat. no. P36935).

- DAPI (Sigma cat. no. D9542)

- Finepoint Forceps (Rubis Switzerland cat.no.1K920)

- Microscope glass slides.

\subsection{Setup and reagents}

4.2.1. PBS Tween 20 solution (PBST): add 0.2\% (V:V) of Tween 20 solution to $1 \mathrm{X}$ PBS solution (see section 1.2.7), mix vigorously to complete dissolution and keep at $4^{\circ} \mathrm{C}$. Bring at room temperature before use. 
4.2.2. Blocking solution: add 5\% of Normal Goat Serum (NGS) to the PBST solution.

210 Prepare the day of use.

211

212

213

214

215

216

217

218

219

220

221

222

223

224

225

226

227

228

229

230

\subsection{Method}

Incubate fixed cells in $250 \mu \mathrm{L}$ of blocking solution for one hour under gentle agitation at room temperature $\left(20-22^{\circ} \mathrm{C}\right)$. Then remove the blocking solution by aspiration and incubate $2 \mathrm{~h}$ at $\mathrm{RT}$ or overnight at $4^{\circ} \mathrm{C}$ with primary antibodies in blocking solution $(250 \mu \mathrm{L} /$ well $)$ under gentle agitation. Wash three times in PBST and incubate for two hours protected from light with specific secondary antibodies diluted in blocking solution (Table 1) $(250 \mu \mathrm{L} /$ well). Wash three times in PBST, incubate 5 minutes in DAPI solution $(1 \mu \mathrm{g} / \mathrm{mL}$ in PBS) for nuclei staining and then wash three times in PBS followed by 1 wash in MilliQ $\mathrm{H}_{2} \mathrm{O}$. Remove coverslips from wells with finepoint Forceps, let coverslips dry completely at room temperature protected from light and mount on glass slides with ProLong ${ }^{\mathrm{TM}}$ Gold Antifade mounting medium. Keep for up to one year at $-20^{\circ}$ protected from light.

\section{Confocal microscope}

Images were acquired with a laser-scanning confocal microscope Leica SP5 using 63X /NA 1.4 oil immersion lens and X5 numerical zoom. The Pinhole was adjusted to 1 airy Unit and the gain was adjusted without offset for each filter on a specific scanning plan allowing specific acquisition without saturation. Image acquisition was performed according to Nyquist parameters in $X Y$ with an average frame of 3 in a sequential scan mode to avoid cross talk between different wavelengths. Z-stacks were obtained by scanning the whole neuron thickness with step of $1 \mu \mathrm{m}$ in $\mathrm{z}$.

\section{Image analysis with ICY open source software}


Confocal images were analyzed with ICY software (http://icy.bioimageanalysis.org/).

232

Quantification was performed on a single plane extracted from a z-stack. The analysis combined two sequential steps. The first one consists in isolating each neuron to define regions of interest (ROI). The second one involves the detection of the spots in each channel and the determination of the amount of co-localisation in each ROI.

\subsection{ROI definition}

Each neuron was carefully delineated using the "free-hand area" tool. This initial ROI is filled with the "fill holes in ROI" plugin to define the total cell area (ROI total). ROIs were then processed to generate two ROIs corresponding to the cell periphery and the cytoplasm (detailed protocol available online http://icy.bioimageanalysis.org/). Based on staining in basal conditions, we estimated that most of the plasma membrane staining was found over an 8 pixels thickness. Therefore, we automatically eroded with the "Erode ROI" plugin the ROI total by 8 pixels and subtracted this new ROI (ROI cyto) to ROI total to obtain a ROI corresponding to the cell periphery (ROI peri).

\subsection{Spots detection and co-localisation}

To detect specific signal in each ROI, we used the "spot detector" plugin which rely on the wavelet transform algorithm [11]. By carefully setting the sensitivity threshold and the scale of objects to detect, it allows detection of spots even in images with low signal to noise ratio. In our conditions, the sensitivity threshold was fixed between 50 and $60 \%$ and the scale of objects set at 2 (pixel size 3) for mu and delta receptors. Once parameters were defined, images were images were processed with the tool "protocol" in Icy which is a graphical interface for automated image processing. Data including the number of spots detected in each channel and ROIs, the number of co-localized objects and the ROI area were 
automatically collected in excel files. Objects were considered co-localized if the distance of

their centroid was equal to or less than 3 pixels. The protocol is available online

(NewColocalizer with binary and excel output v1_batch.xml). To obtain histograms we calculated object densities for each receptor reported to the surface of each ROI. Membrane to cytoplasm density ratios were calculated to illustrate the subcellular distribution of each receptor. The extend of co-localisation was calculated according to the following formula for each ROI $\left[\%\right.$ colocalisation $\left.=100 X\left(\frac{\text { colocalised } M O P \text { and DOP objects }}{\sum(\text { detected } M O P \text { and DOP objects })}\right)\right]$.

\section{Statistical analysis}

Statistical analyses were performed with Graphpad Prism V7 software (GraphPad, San

Diego, CA). Normality of the distributions and homogeneity of the variances were checked before statistical comparison to determine appropriate statistical analysis. In our case, data were not normally distributed and the non-parametric Mann Witney test was used to compare receptor densities in the plasma membrane and cytoplasm in basal conditions or after agonist treatment. The extend of receptor co-localisation was compared using two-way ANOVA with repeated measures followed by post-hoc Sidak's test for multiple comparisons. Basal group was compared to agonist treated group (first factor) within cytoplasm and plasma membrane localisation (second factor).

\section{RESULTS AND DISCUSSION}

In this chapter, we have presented an optimized method for monitoring the subcellular distribution of endogenous MOP and DOP receptors. To this aim, we combined the use of a genetically modified mouse line co-expressing functional fluorescently tagged receptors, optimized primary neuronal culture protocol and automatic quantitative analysis of confocal 
images with an open source software. Importantly, the image analysis procedure can be easily implemented in any laboratory since data processing does not require extensive mathematical developments or program writing with specialized software.

\section{Individual distribution of MOP and DOP receptors}

High magnification confocal images analyzed as single focal plan revealed discontinuous and punctate distributions for DOP-eGFP and MOP-mCherry that were predominantly located at the cell surface in basal conditions (Figure 2-A). Images also revealed a perinuclear cytoplasmic localization of both receptors that likely correspond to receptor stock in the endoplasmic reticulum. These observations were consistent with data from the literature describing a predominant and membrane localization of DOP [10,12] and MOP [13] but also substantial localization in perikarya [10].

Quantification using the ICY software indicated a higher density of fluorescent objects at the cell surface for both MOP and DOP receptors (Figure 2-B) that was three times higher compared to the cytoplasm (Fig. 2-C). Activation by the MOP-DOP agonist CYM51010 let to the appearance of high intensity punctate structures in the cytoplasmic and a dramatic decrease in the plasma membrane labelling of both receptors (figure 2-A). Accordingly, the ratio corresponding to the density of fluorescent objects density at the plasma membrane compared to the cytoplasm dropped dramatically from 3 to 1 for both MOP and DOP receptors which supports internalization of the two receptors in vesicle-like structures.

These results are in agreement with previous reports using the density of fluorescence to estimate changes in DOPeGFP subcellular distribution in vivo. In these studies, the ratio of fluorescence density between the plasma membrane and cytoplasm was about 1.5 in basal 
conditions and significantly decreased following agonist stimulation $[10,14]$. We therefore

tested our quantification method using images of DOR-eGFP neurons in the hippocampus acquired with similar parameters in confocal microscopy. Using the protocol described above, we found that the density in DOP-eGFP objects under basal conditions was around 3 similar to our results in primary neuronal cultures. We also established that this value corresponded to a ratio in fluorescence density between the plasma membrane and the cytoplasm of about 1.7, similar to previously reported ratios [10]. Moreover, we quantified set of images used in Erbs et al 2016, and calculated a similar increase of about 10\% in DOPeGFP expression at the plasma membrane after chronic morphine treatment in the neurons of the hippocampus [15]. Altogether, these results fully validate the quantification method developed using ICY software.

\section{Co-localization of MOP and DOP receptors and detection of MOP-DOP}

heteromers

Our analysis revealed substantial colocalization of MOP and DOP associated signals under basal conditions. In fact, more than $22 \%$ of the MOP and DOP objects were co-localized within the plasma membrane whereas cytoplasmic colocalization was fairly low (around 10\%) (Figure 2-D). After selective MOP-DOP activation with the agonist CYM51010, the cytoplasmic colocalization was increased by $87 \%$ to reach $18.7 \%$. A two-fold decrease in the

317 percentage of MOP-DOP colocalization within the plasma membrane was also observed after CYM51010 treatment (Figure 2-D). These observations support a view in which MOP and DOP receptors remain associated upon specific activation of the heteromers and undergo common intracellular fate. 
321 It is however important to note, that due to the resolution limit of confocal microscopy,

322 MOP-DOP physical interaction has to be assessed by other experimental approaches such as co-localization in electron microscopy or disruption of the physical contact by a transmembrane peptide. The latter has been successfully performed for MOP-DOP receptors in vivo. Indeed, a peptide corresponding to the MOP TM 1 or to the DOP second intracellular loop in fusion with the cell transduction domain of the human immunodeficiency virus (HIV) TAT protein interfered with MOP-DOP co-immunoprecipitation [16-18]. The recently described proximity ligation assay [19] provides high spatial resolution and represents another attractive option to address physical proximity.

In addition, the resolution of the images did not enable to distinguish the pool of receptors associated to the plasma membrane from receptors located in the sub-membrane compartment which represents another limitation of our analysis. Total internal reflection fluorescence microscopy (TIRFM) would be required to differentiate the two compartments and precisely monitor the first steps in receptor internalization.

MOPmcherry-DOPeGFP co-localisation studies could also be combined with identification of the intracellular compartments using specific antibodies. This would enable for fine mapping of the receptors in the vesicular structures and cellular compartments and would provide detailed information on the intracellular fate of MOP-DOP heteromers.

\section{CONCLUSION}

Double knock-in mice represent unique tools to investigate endogenous MOP-DOP neuroanatomical distribution but also to explore functional dynamics in physio-pathological conditions. As described here, the use of double fluorescent knock-in mice combined with 
confocal imaging and ICY software analyses enables easy quantification of receptor subcellular distribution and co-localisation, hence specific MOP-DOP trafficking. Importantly, the field of application of the method described here is not restricted to the study of MOPDOP heteromers but can be applied to address the heteromerization of any other pair of GPCRs. Moreover, the analysis with the ICY software is not restricted to the study of receptors but is widely applicable to monitor the co-localisation of any two objects detected independently and can be applied to any type of high-resolution images.

\section{NOTES}

1- Cell dissociation is a critical step; if the trituration is too gentle, the tissue will not dissociate, if too vigorous cells will break.

2- Because re-expression of DOP-eGFP and MOP-mCherry is only detectable from day in vitro (DIV) 10 in primary neurons, pharmacological treatments should be performed between DIV12 and DIV15 to ensure full expression of the receptors.

3- Paraformaldehyde fixation decreases the fluorescence intensity of eGFP and mCherry and amplification by immunostaining is recommended. The DOP-eGFP construct can also be used for ex vivo real-time imaging of receptor internalization by confocal microscopy [10]. However, due to the low expression level of endogenous MOP receptors and their weak expression at the plasma membrane in basal conditions, real-time monitoring of MOP-mcherry remains presently below the detection limit.

\section{ACKNOWLEDGMENTS}


The authors would like to acknowledge the financial support of the Fondation pour la

Recherche Médicale (LPA20140129364). L. Derouiche was the recipient of an IDEX postdoctoral fellowship of the University of Strasbourg.

\section{CONFLICT OF INTEREST}

The authors declare no conflict of interest

\section{REFERENCES}

1. Charbogne P, Kieffer BL, Befort K (2014) 15 years of genetic approaches in vivo for addiction research: Opioid receptor and peptide gene knockout in mouse models of drug abuse. Neuropharmacology 76 Pt B:204-217. doi:10.1016/j.neuropharm.2013.08.028

2. Pasternak GW (2014) Opioids and their receptors: Are we there yet? Neuropharmacology 76 Pt B:198-203. doi:10.1016/j.neuropharm.2013.03.039

3. Civelli O (2008) The orphanin FQ/nociceptin (OFQ/N) system. Results Probl Cell Differ 46:1-25. doi:10.1007/400_2007_057

4. Ong EW, Cahill CM (2014) Molecular Perspectives for mu/delta Opioid Receptor Heteromers as Distinct, Functional Receptors. Cells 3 (1):152-179. doi:10.3390/cells3010152

5. Gendron L, Mittal N, Beaudry H, Walwyn W (2015) Recent advances on the delta opioid receptor: from trafficking to function. $\mathrm{Br} J$ Pharmacol 172 (2):403-419. doi:10.1111/bph.12706

6. Gomes I, Ijzerman AP, Ye K, Maillet EL, Devi LA (2011) G protein-coupled receptor heteromerization: a role in allosteric modulation of ligand binding. Mol Pharmacol 79 (6):1044-1052. doi:10.1124/mol.110.070847 
heteromers as novel therapeutic targets: IUPHAR review 10. Br J Pharmacol 171 (18):41554176. doi:10.1111/bph.12798

8. Massotte D (2015) In vivo opioid receptor heteromerization: where do we stand? Br J

Pharmacol 172 (2):420-434. doi:10.1111/bph.12702

9. Erbs E, Faget L, Scherrer G, Matifas A, Filliol D, Vonesch JL, Koch M, Kessler P,

Hentsch D, Birling MC, Koutsourakis M, Vasseur L, Veinante P, Kieffer BL, Massotte D

(2015) A mu-delta opioid receptor brain atlas reveals neuronal co-occurrence in subcortical networks. Brain Struct Funct 220 (2):677-702. doi:10.1007/s00429-014-0717-9

Dierich A, Vonesh JL, Gaveriaux-Ruff C, Kieffer BL (2006) Knockin mice expressing compartmentalization. J Comp Neurol 440 (1):65-84

fluorescent delta-opioid receptors uncover $G$ protein-coupled receptor dynamics in vivo. Proc Natl Acad Sci U S A 103 (25):9691-9696. doi:10.1073/pnas.0603359103

11. Olivo-Marin JC (2002) Extraction of spots in biological images using multiscale products. Pattern Recogn 35 (9):1989-1996. doi:Pii S0031-3202(01)00127-3 Doi 10.1016/S00313203(01)00127-3

12. Cahill CM, McClellan KA, Morinville A, Hoffert C, Hubatsch D, O'Donnell D, Beaudet A (2001) Immunohistochemical distribution of delta opioid receptors in the rat central nervous system: evidence for somatodendritic labeling and antigen-specific cellular

13. Trafton JA, Abbadie C, Marek K, Basbaum AI (2000) Postsynaptic signaling via the [mu]-opioid receptor: responses of dorsal horn neurons to exogenous opioids and noxious stimulation. J Neurosci 20 (23):8578-8584 
Decossas M, Koch M, Kessler P, Vonesch JL, Schwab Y, Kieffer BL, Massotte D (2012) In vivo visualization of delta opioid receptors upon physiological activation uncovers a distinct internalization profile. J Neurosci 32 (21):7301-7310. doi:10.1523/JNEUROSCI.0185-12.2012

Impact of chronic morphine on delta opioid receptor-expressing neurons in the mouse hippocampus. Neuroscience 313:46-56. doi:10.1016/j.neuroscience.2015.10.022

Wang Q, Lu YJ, Bao L, Zhang X (2011) Facilitation of mu-opioid receptor activity by preventing delta-opioid receptor-mediated codegradation. Neuron 69 (1):120-131. doi:10.1016/j.neuron.2010.12.001

17. Kabli N, Martin N, Fan T, Nguyen T, Hasbi A, Balboni G, O'Dowd BF, George SR

(2010) Agonists at the delta-opioid receptor modify the binding of micro-receptor agonists to

the micro-delta receptor hetero-oligomer. $\mathrm{Br} \quad \mathrm{J}$ Pharmacol 161 (5):1122-1136.

18. Xie WY, He Y, Yang YR, Li YF, Kang K, Xing BM, Wang Y (2009) Disruption of

Cdk5-associated phosphorylation of residue threonine-161 of the delta-opioid receptor: impaired receptor function and attenuated morphine antinociceptive tolerance. J Neurosci 29

(11):3551-3564. doi:10.1523/JNEUROSCI.0415-09.2009

19. Dasiel O. Borroto-Escuela BH, Miles Woolfenden, Luca Pinton, Antonio Jiménez- 


\section{TABLE 1: Primary and secondary antibodies}

\begin{tabular}{|l|l|l|l|}
\hline Antigen & Antibody & Supplier reference & Dilution \\
\hline eGFP & Chicken IgY & AVES-GFP1020 & $1 / 1000$ \\
\hline mCherry & Rabbit IgG & Clontech-632496 & $1 / 1000$ \\
\hline Chicken IgY & Goat anti chicken Alexa Fluor 488 & Molecular Probes-A11039 & $1 / 2000$ \\
\hline Rabbit IgG & Goat anti rabbit Alexa Fluor 594 & Molecular Probes-A11012 & $1 / 2000$ \\
\hline
\end{tabular}

\section{FIGURE LEGENDS}

Figure 1: Double Knock-in DOP-eGFP/MOP-mCherry mouse engineering. A)

Construction of the DOP-eGFP mouse. A cDNA sequence corresponding to the eGFP cDNA, and the FRT flanked neomycine (neo) cassette was inserted by homologous recombination (HR) into the Oprd1 locus. HR was followed Cre recombinase treatment in ES cells. B) locus. HR was followed by FRT recombinase treatment in ES cells. (C) Double knock-in mice were obtained by crossing homozygote DOP-eGFP and MOP-mCherry mice.

Figure 2: MOP-DOP heteromer visualisation and quantification. A) Representative 
or after treatment during 60 minutes with the MOP-DOP agonist CYM51010 400nM. Scale

447 bar: $5 \mu \mathrm{m}$. B) Image quantification with ICY software illustrating each receptor distribution.

448 In basal conditions, MOP and DOP densities are significantly higher in the plasma

449 membrane compared to cytoplasm. Mann Witney test, ${ }^{*}$ p-value $<0.05$. CYM51010 treatment

450 for 60 minutes led to changes in receptor subcellular distribution with no more statistical

451 difference in receptor densities between the plasma membrane and cytoplasm (Mann Witney

452 test, $\mathrm{p}$-value $>0.05)$. C) Changes in receptors distribution. Plasma membrane to cytoplasm

453 ratio of MOP or DOP spots densities in basal conditions or after with 400nM CYM51010 for

45460 minutes reflects receptor redistribution. Mann Witney test, ${ }^{* * * *}$-value $<0.001 ;{ }^{* * * *} p$-value

$455<0.0001$. D) Quantification of MOP-DOP co-localisation upon agonist activation with 400nM

456 CYM51010 for 60 minutes reveals MOP-DOP co-internalisation. Two-way ANOVA with

457 repeated measures, post-hoc Sidak's test. * $\mathrm{p}$-value $<0.05$ for basal cytoplasm vs CYM51010

458 cytoplasm; ** p-value $<0.01$ for basal membrane vs CYM51010 membrane. \#\#\# p-value $<0.001$

459 for basal membrane $v$ s basal cytoplasm; NS: p-value >0.05 for CYM51010 membrane vs

460 CYM51010 cytoplasm. 
A

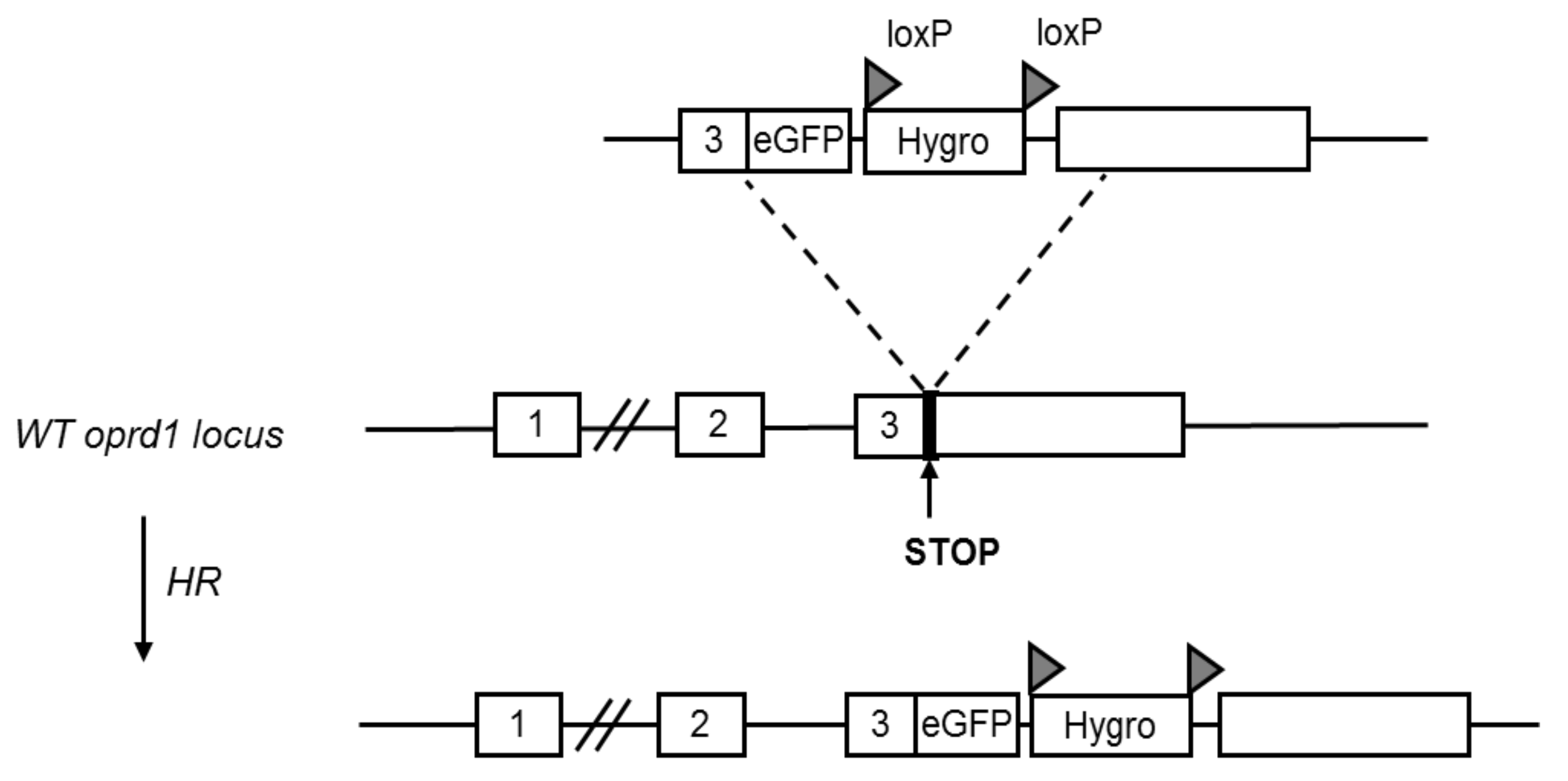

Cre recombinase

Targeted oprd 1 locus

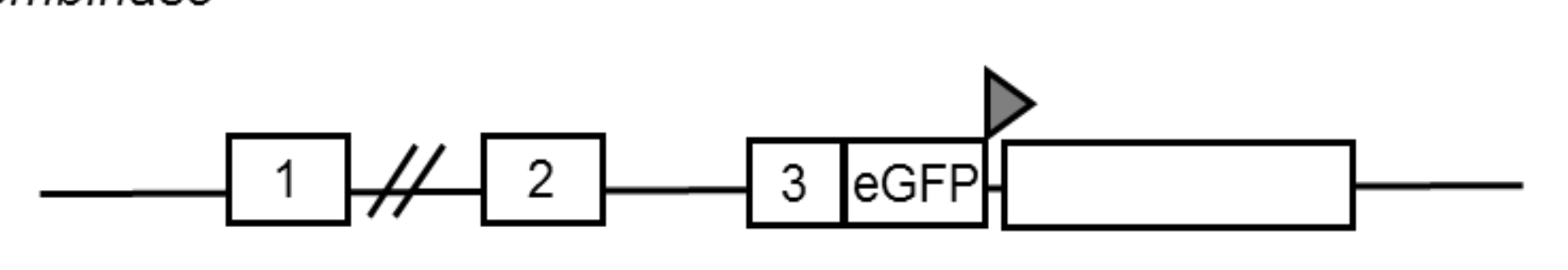

B

WT oprm1 locus
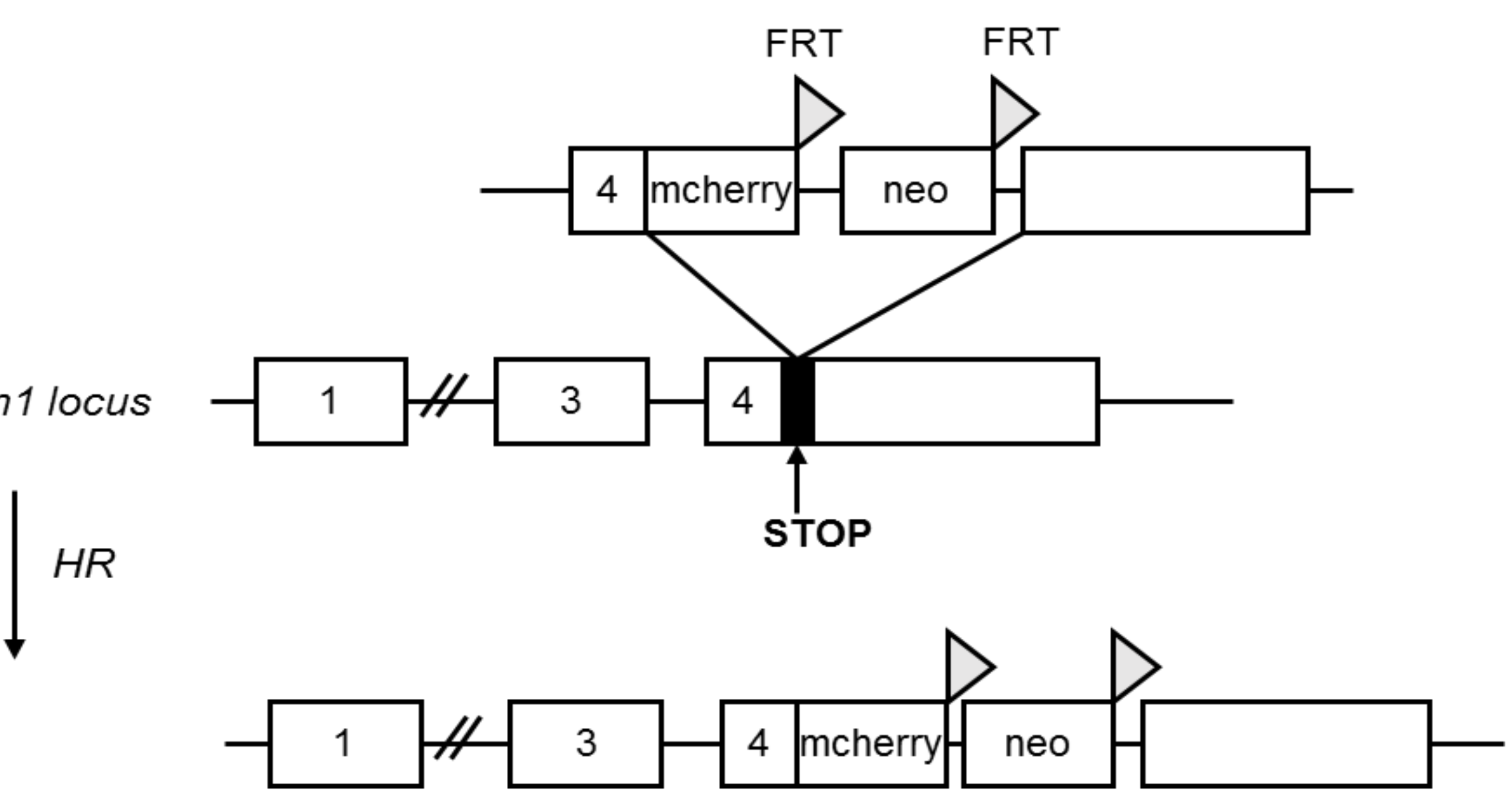

FLP recombinase

Targeted oprm1

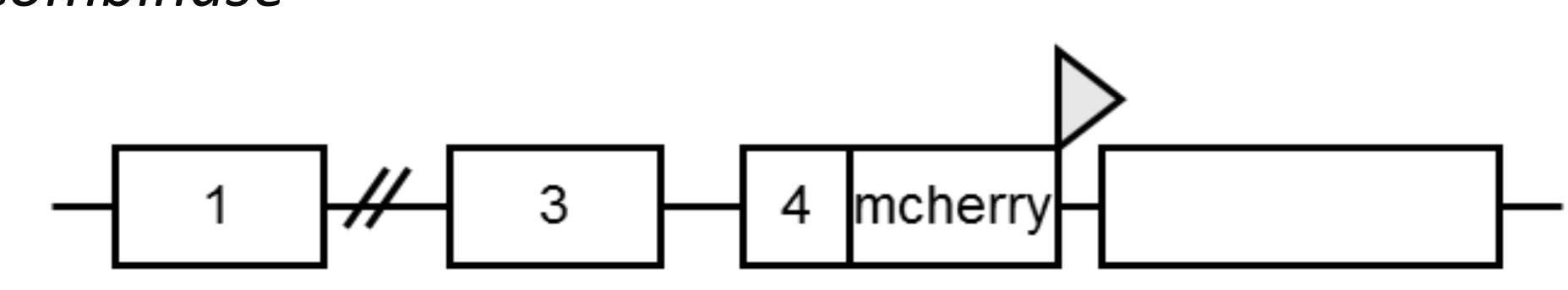

C DOP-eGFP/

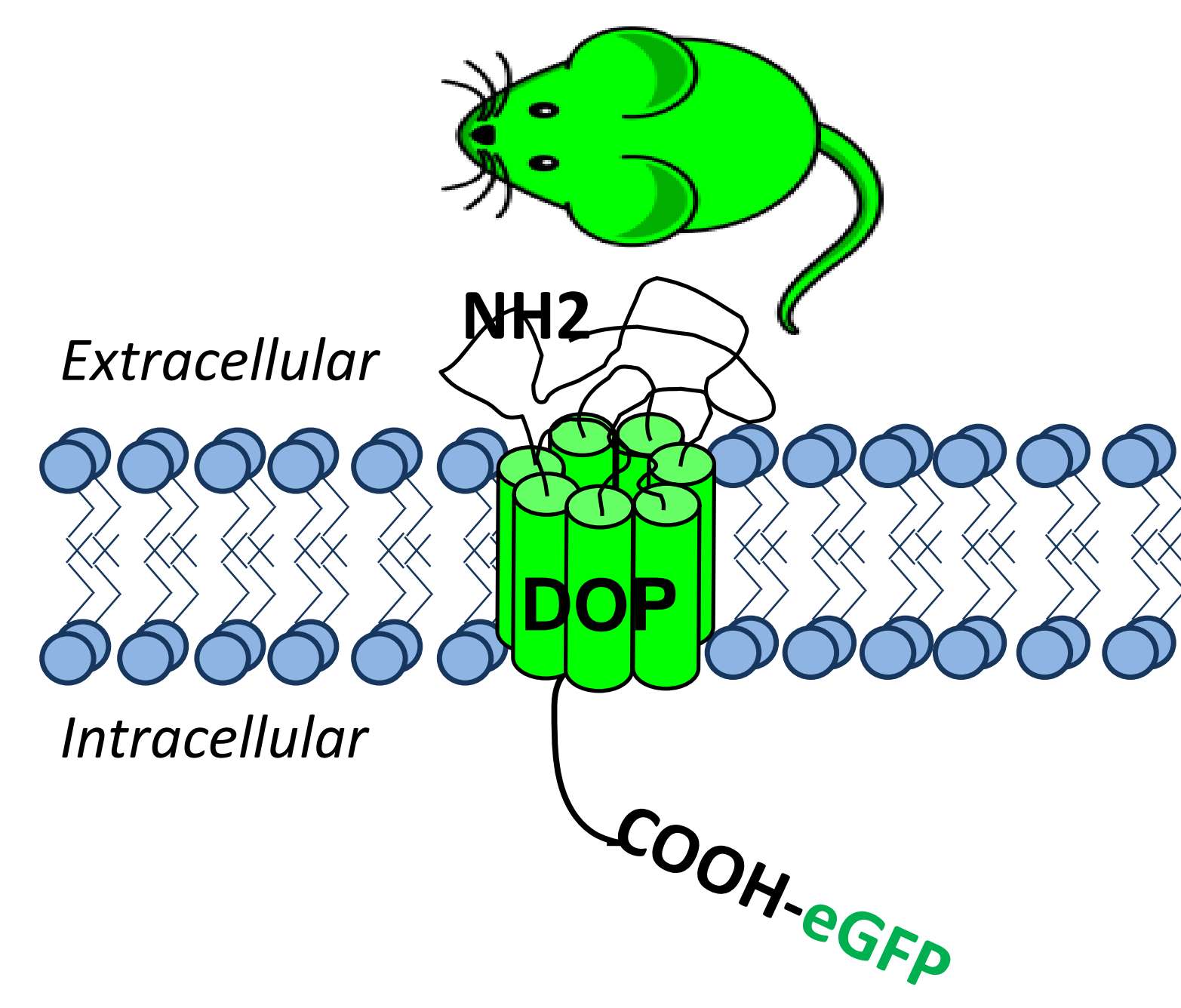

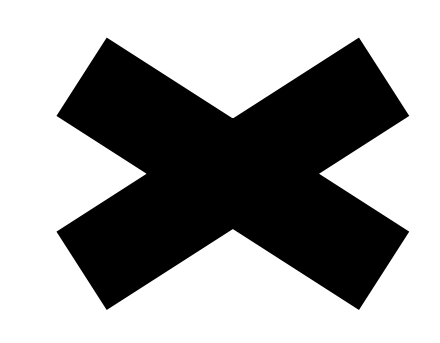

E DOP-eGFP/MOP-mCherry

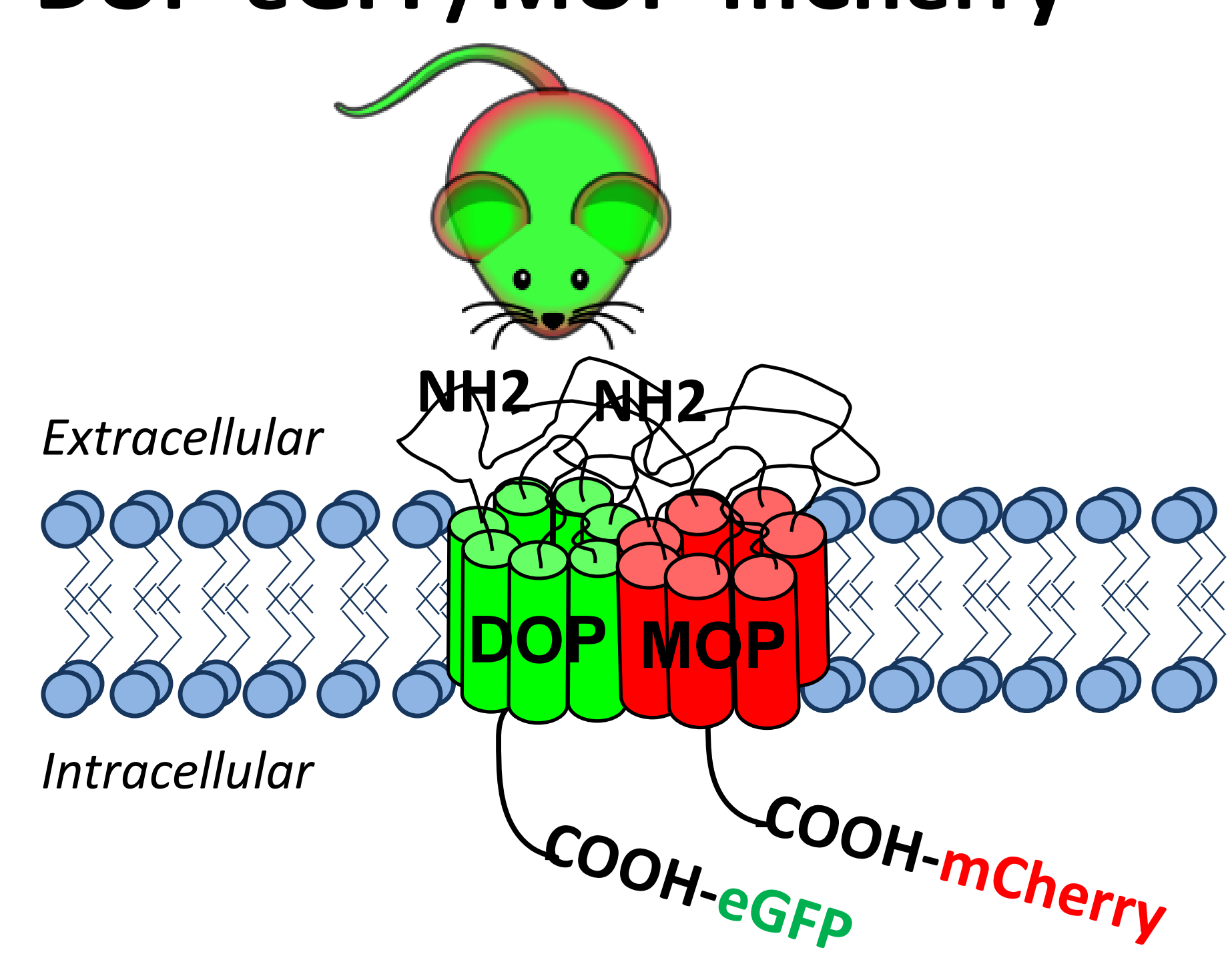

D MOP-mCherry

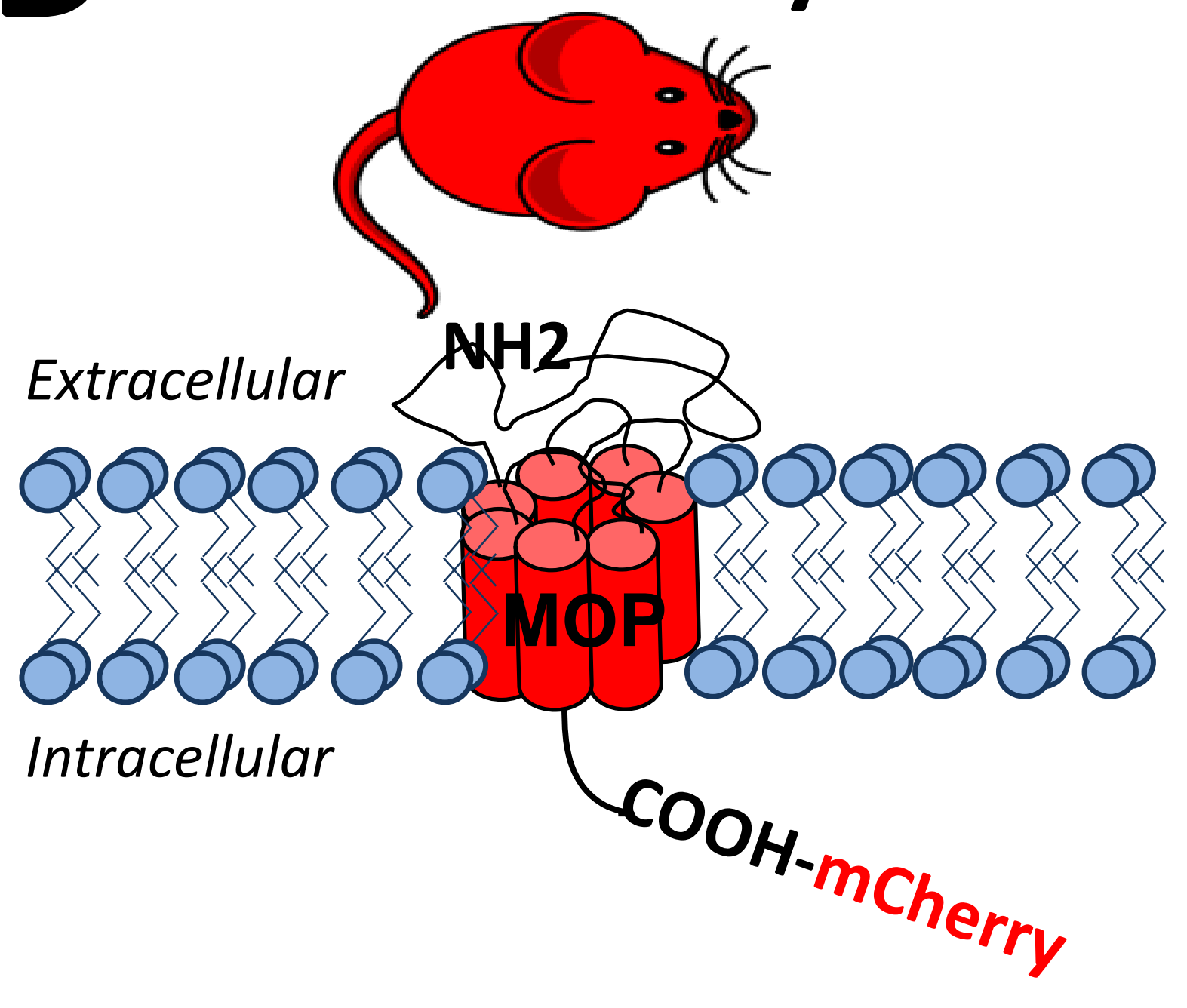


